\title{
Comparison of combined leflunomide and low-dose corticosteroid therapy with full-dose corticosteroid monotherapy for progressive IgA nephropathy
}

\author{
Lulin Min ${ }^{1}$, Qin Wang ${ }^{1}$, Liou Cao ${ }^{1}$, Wenyan Zhou ${ }^{1}$, Jiangzi Yuan ${ }^{1}$, Minfang Zhang $^{1}$, \\ Xiajing Che ${ }^{1}$, Shan Mou ${ }^{1}$, Wei Fang ${ }^{1}$, Leyi Gu${ }^{1}$, Mingli Zhu ${ }^{1}$, Ling Wang ${ }^{1}$, Zanzhe \\ Yu ${ }^{1}$, Jiaqi Qian ${ }^{1}$ and Zhaohui $\mathbf{N i}^{1}$ \\ ${ }^{1}$ Department of Nephrology, Molecular Cell Lab for Kidney Disease, Ren Ji Hospital, School of Medicine, Shanghai Jiao Tong \\ University, Shanghai, China \\ Correspondence to: Zhaohui Ni, email: profnizh@126.com \\ Keywords: IgA nephropathy, leflunomide, proteinuria, corticosteroids, renal survival \\ Received: January 28, $2017 \quad$ Accepted: March 06, $2017 \quad$ Published: March 22, 2017 \\ Copyright: Min et al. This is an open-access article distributed under the terms of the Creative Commons Attribution License 3.0 (CC BY 3.0), \\ which permits unrestricted use, distribution, and reproduction in any medium, provided the original author and source are credited.
}

\section{ABSTRACT}

IgA nephropathy is the most common primary glomerulonephritis and one of the leading causes of end-stage renal disease. We performed a randomized, controlled, prospective, open-label trial to determine whether leflunomide combined with low-dose corticosteroid is safe and effective for the treatment of progressive IgA nephropathy, as compared to full-dose corticosteroid monotherapy. Biopsyproved primary IgA nephropathy patients with an estimated glomerular filtration rate $\geq 30 \mathrm{ml} / \mathrm{min} / 1.73 \mathrm{~m}^{2}$ and proteinuria $\geq 1.0 \mathrm{~g} / 24 \mathrm{~h}$ were randomly assigned to receive leflunomide+low-dose corticosteroid (leflunomide group; $n=40$ ) or fulldose corticosteroid (corticosteroids group; $n=45$ ). The primary outcome was renal survival; secondary outcomes were proteinuria and adverse events. After 12 months of treatment and an average follow-up of 88 months, $11.1 \%$ vs. $7.5 \%$ of patients reached end-stage renal disease and $20 \%$ versus $10 \%$ of patients had a $\geq 50 \%$ increase in serum creatinine in the corticosteroids and leflunomide groups, respectively. Kaplan-Meier analysis did not reveal a between-group difference in these outcomes. Decreases in 24-hour proteinuria were similar in the two groups during the treatment period, but a more marked reduction was observed during follow-up in the leflunomide group. Although the incidence of adverse events was similar in the two groups, serious adverse events were observed only in the corticosteroid group. Thus, leflunomide combined with low-dose corticosteroid is at least as effective as corticosteroid alone for the treatment of progressive IgA nephropathy, and showed a greater reduction of proteinuria during long-term follow-up and fewer severe adverse events.

\section{INTRODUCTION}

IgA nephropathy (IgAN) is the most common primary glomerulonephritis (GN) and one of the leading causes of end-stage renal disease (ESRD) worldwide [12]. The incidence of IgAN is particularly high in the East Asian population, accounting for $>40 \%$ of kidney biopsy specimens obtained from patients with primary GN in China or Japan [1]. IgAN is recognized as a chronically progressive disease. Among patients with biopsy-proven IgAN, 15\%-20\% reach ESRD within 10 years and 20\%-
$40 \%$ within 20 years [3]. At present, treatment options for IgAN are still very limited. Disease management mainly consists of controlling blood pressure and lipid levels and reducing proteinuria by ACEI or ARB, together with other supportive treatment. In addition, the 2012 KDIGO GN Guidelines recommend that patients with persistent proteinuria $(\geq 1 \mathrm{~g} / \mathrm{d}$ and eGFR $>50 \mathrm{ml} / \mathrm{min}$ per $1.73 \mathrm{~m}^{2}$ ) despite 3-6 months of optimized supportive care should receive a 6-month course of CS therapy [4]. However, the long-term use of CS is associated with many side effects, including abnormal glucose metabolism, 
osteoporosis, etc. Some patients with IgAN may also develop CS resistance or dependence, or relapse after withdrawal of steroids. Therefore, several clinical trials have been performed to determine whether combination therapy of steroids and immunosuppressive medications such as cyclophosphamide [5-6], azathioprine [7-8], mycophenolate mofetil [9-14], tacrolimus [15], and mizoribine [16] could provide better renal outcomes and fewer side effects. However, the results were inconsistent [17]. Leflunomide (LEF), an immunosuppressive agent that inhibits the synthesis of pyridines, has been widely used in rheumatoid and renal diseases in recent years [1821]. Several randomized trials evaluated the efficacy of LEF in the treatment of IgAN. The results suggested that LEF reduced the amount of proteinuria and ameliorated renal function deterioration, with only mild side effects [22-24]. Yet, randomized long-term follow-up studies are lacking. Therefore, we conducted here a randomized, controlled, prospective, open-label trial with long-term follow-up to determine whether LEF plus low-dose CS is safe and effective for the treatment of progressive IgAN, as compared to full-dose CS alone.

\section{RESULTS}

In total, 90 patients with IgAN were randomly assigned to one of two groups: 1) CS (full-dose prednisone); 2) LEF (LEF plus low-dose prednisone). Five patients dropped off before treatment. Of the remaining 85 patients, 45 were assigned to the CS group and 40 to the LEF group. 80 patients (43 patients in the CS group and 37 patients in the LEF group) completed the 12-month treatment and were followed up for a median period of 88 months (Figure 1).

\section{Baseline characteristics}

Table 1 shows the baseline characteristics of the groups. Age, sex, systolic blood pressure (SBP), diastolic blood pressure (DBP), 24-hour proteinuria, serum creatinine ( $\mathrm{SCr}$ ) levels, eGFR (by CKD-EPI equation), histological characteristics, and other pre-treatment clinical biochemical indexes were comparable between groups.

\section{Outcome of treatment}

After 12 months of treatment, proteinuria (g/24h) levels decreased significantly in both groups. In the CS group, it decreased from 2.16 (IQR 1.36 to 3.5) at baseline to 0.83 (IQR 0.32 to 1.94 ) at 6 months, and to 0.65 (IQR 0.17 to 1.46$)$ at 12 months $(p<0.01)$. In the LEF group, proteinuria decreased from 1.94 (IQR 1.21 to 2.87) at baseline to 0.54 (IQR 0.15 to 1.15 ) at 6 months, and to 0.29 (IQR 0.08 to 0.69$)$ at 12 months $(p<0.01)$. Whereas baseline and 6-month values were similar between groups, 12-month proteinuria levels in the LEF group were significantly lower than in the CS group $(p<0.05$; Table 2).

Renal function was stable in both groups through the 12-month treatment period, as indicated by SCr and eGFR,

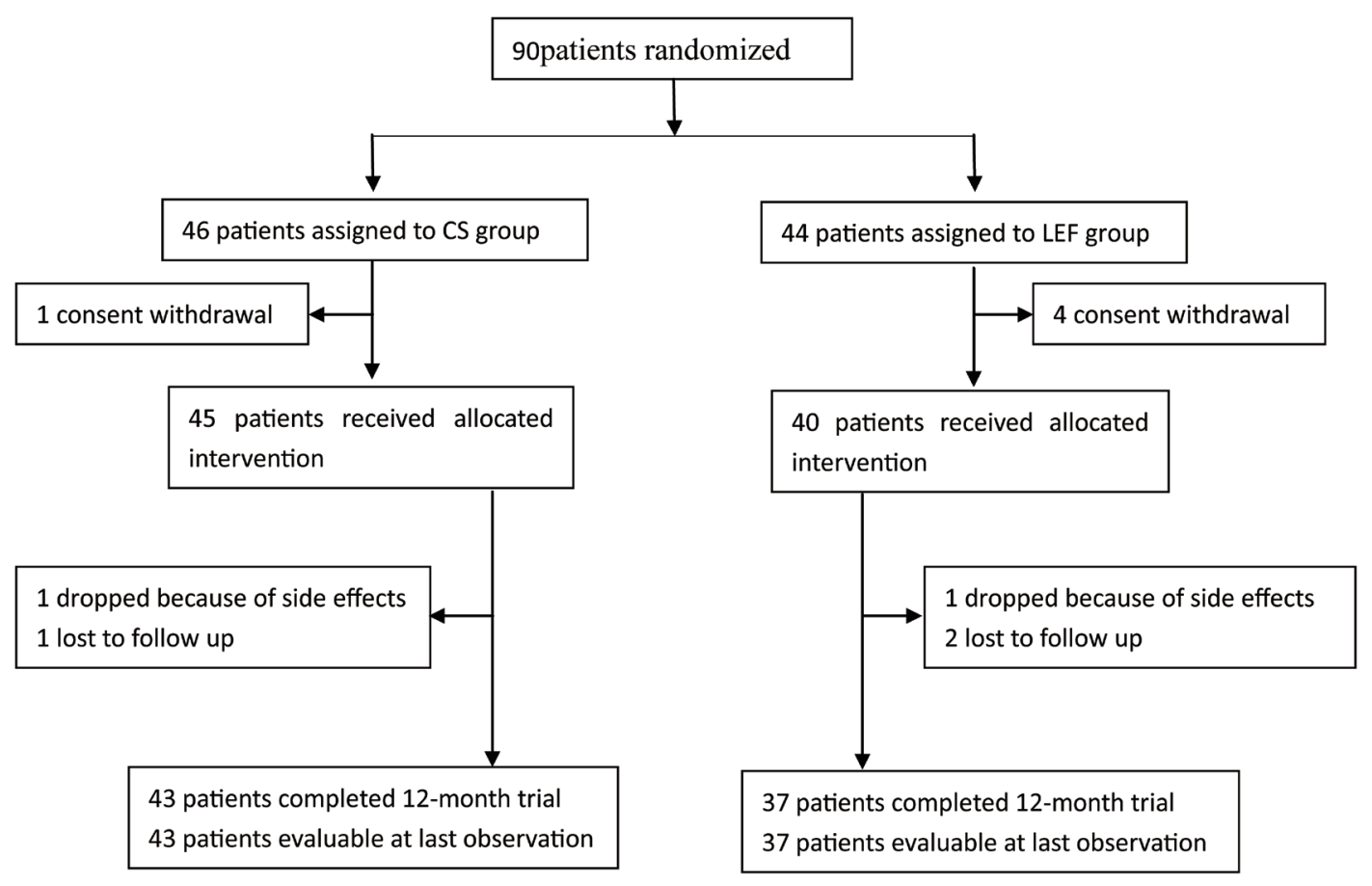

Figure 1: Patient enrollment and follow-up. 
Table 1: Baseline clinical and histological characteristics of the study subjects

\begin{tabular}{|c|c|c|c|}
\hline Characteristic & $\begin{array}{c}\text { CS group } \\
(n=45)\end{array}$ & $\begin{array}{l}\text { LEF group } \\
(n=40)\end{array}$ & $p$ value \\
\hline Age (years) & $36.60 \pm 11.53$ & $36.90 \pm 10.49$ & 0.901 \\
\hline Sex (male) & 22 & 14 & 0.272 \\
\hline $\mathrm{SBP}(\mathrm{mmHg})$ & $121.69 \pm 16.78$ & $122.15 \pm 12.98$ & 0.889 \\
\hline DBP (mmHg) & $79.29 \pm 11.48$ & $79.05 \pm 9.86$ & 0.919 \\
\hline $\mathrm{SCr}(\mu \mathrm{mol} / \mathrm{L})$ & $95.14 \pm 31.55$ & $92.43 \pm 33.99$ & 0.705 \\
\hline $\mathrm{UA}(\mu \mathrm{mol} / \mathrm{L})$ & $380.08 \pm 87.44$ & $356.77 \pm 104.54$ & 0.266 \\
\hline UPE $(g / 24 h)$ & $1.78(1.31,3.49)$ & $1.91(1.18,2.88)$ & 0.420 \\
\hline ALB (mmol/L) & $37.73 \pm 4.10$ & $38.25 \pm 4.13$ & 0.569 \\
\hline GLU & $5.00 \pm 0.53$ & $4.92 \pm 0.57$ & 0.493 \\
\hline $\mathrm{Hb}$ & $133.89 \pm 16.81$ & $131.55 \pm 18.12$ & 0.542 \\
\hline $\operatorname{ALT}(\mathrm{U} / \mathrm{L})$ & $19.94 \pm 11.07$ & $24.21 \pm 21.40$ & 0.249 \\
\hline AST (U/L) & $19.85 \pm 5.86$ & $22.02 \pm 11.26$ & 0.067 \\
\hline $\mathrm{TG}(\mathrm{mmol} / \mathrm{L})$ & $1.90 \pm 0.93$ & $1.97 \pm 1.12$ & 0.737 \\
\hline $\mathrm{TC}(\mathrm{mmol} / \mathrm{L})$ & $5.19 \pm 1.09$ & $5.10 \pm 1.32$ & 0.730 \\
\hline LDL $(\mathrm{mmol} / \mathrm{L})$ & $3.16 \pm 0.80$ & $3.11 \pm 0.96$ & 0.799 \\
\hline $\mathrm{eGFR}\left(\mathrm{ml} / \mathrm{min} / 1.73 \mathrm{~m}^{2}\right)$ & $84.26 \pm 29.05$ & $84.10 \pm 25.55$ & 0.979 \\
\hline segmental or global glomerulosclerosis/ total glomeruli (\%) & $39.69 \pm 20.79$ & $40.13 \pm 26.32$ & 0.932 \\
\hline interstitial fibrosis and chronic inflammation (score) & $1.58 \pm 0.71$ & $1.56 \pm 0.67$ & 0.919 \\
\hline tubule atrophy (score) & $1.52 \pm 0.67$ & $1.51 \pm 0.71$ & 0.972 \\
\hline inflammatory cell infiltration (score) & $2(1,2)$ & $1(1,2.88)$ & 0.555 \\
\hline crescentic glomeruli/ total glomeruli (\%) & $4(0,11.5)$ & $5(0,10)$ & 0.859 \\
\hline Follow-up (months) & $89.12 \pm 22.61$ & $87.22 \pm 21.24$ & 0.701 \\
\hline
\end{tabular}

Values are presented as means \pm standard deviation (SD) if the variables showed a normal distribution and as medians (IQR) if the variables did not show a normal distribution; $\mathrm{n}(\%)$ was used for the categorical variables.

SBP: systolic blood pressure; DBP: diastolic blood pressure; SCr: serum creatinine; UA: serum uric acid; UPE: Urine protein excretion; ALB: serum albumin; GLU: glucose; Hb: hemoglobin; ALT: alanine transaminase; TG: triglyceride; TC: cholesterol; LDL: low density lipoprotein; eGFR: estimated glomerular filtration rate; AST: aspartate aminotransferase.

which were no different between groups (Table 2).

At the end of treatment, a total of 31 patients (68.9\%) achieved remission (14 CR, $17 \mathrm{PR})$ in the CS group, whereas a total of 27 patients $(67.5 \%)$ achieved remission (16 CR, $11 \mathrm{PR}$ ) in the LEF group. However, among the remission patients, 5 patients in the CS group and 4 in the LEF group experienced relapse within one year after withdrawal of drugs. There was no difference in the total remission and relapse rates between the two groups.

\section{Renal survival}

No statistically significant differences in primary outcomes were noted between groups. However, there was a trend towards better outcomes in the LEF group. The rate of ESRD was $11.1 \%(5 / 45)$ in the CS group vs. $7.5 \%(3 / 40)$ in the LEF group. A 50\% increase in $\mathrm{SCr}$ from baseline occurred in $20 \%$ of patients $(9 / 45)$ in the CS group vs. $10 \%$ of patients (4/40) in the LEF group. The cumulative probabilities of event-free survival for the primary outcomes were calculated using the Kaplan-Meier method. No significant between-treatment differences were observed (Figure 2).

\section{Proteinuria}

The results summarized in Table 2 show, as described above, that 24-hour proteinuria levels decreased significantly in both groups during the 12-month treatment period. After withdrawal of drugs, there was no rebound in either group during the follow-up period (Figure 3). The 24-hour proteinuria levels in the CS group were 0.53 (IQR 0.11 to 1.32 ) at 24 months, 0.61 (IQR 0.18 to 1.68 ) at 36 months, 0.62 (IQR 0.21 to 1.48 ) at 48 months, and 0.96 (IQR 0.32 to 1.75 ) at 60 months. Meanwhile, in the LEF group proteinuria values were 0.16 (IQR 0.04 to 0.58 ) at 24 months, 0.2 (IQR 0.1 to 0.95 ) at 36 months, 0.23 (IQR 0.13 to 1.31 ) at 48 months, and 0.27 (IQR 0.15 to 1.03 ) at 60 months. Significantly lower urine protein excretion rates were observed at 24, 36, and 60 months in the LEF group. $(P<0.05$; Figure 3$)$. 
(a) Time to ESRD

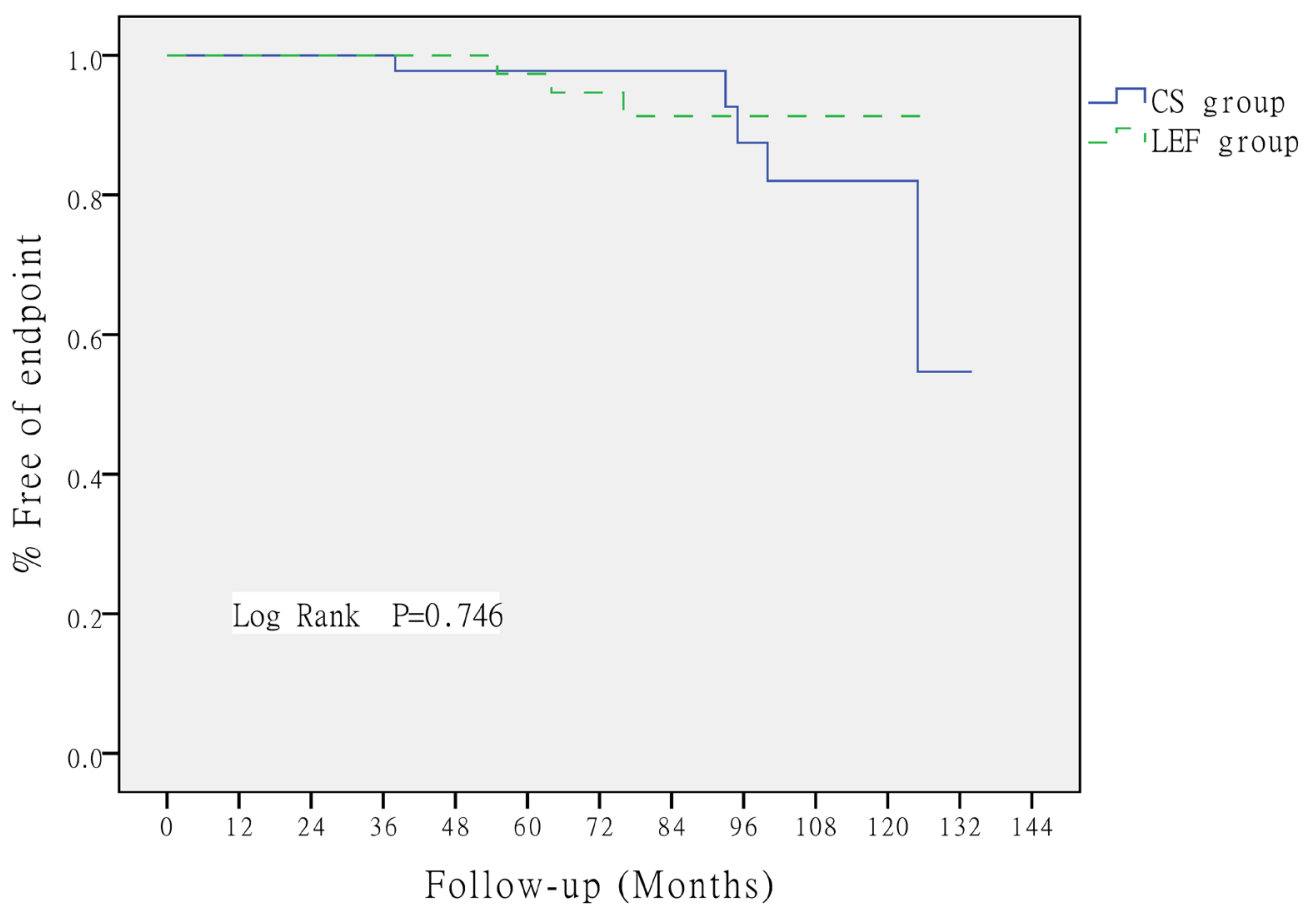

(b) Time to a $50 \%$ increase in serum creatinine

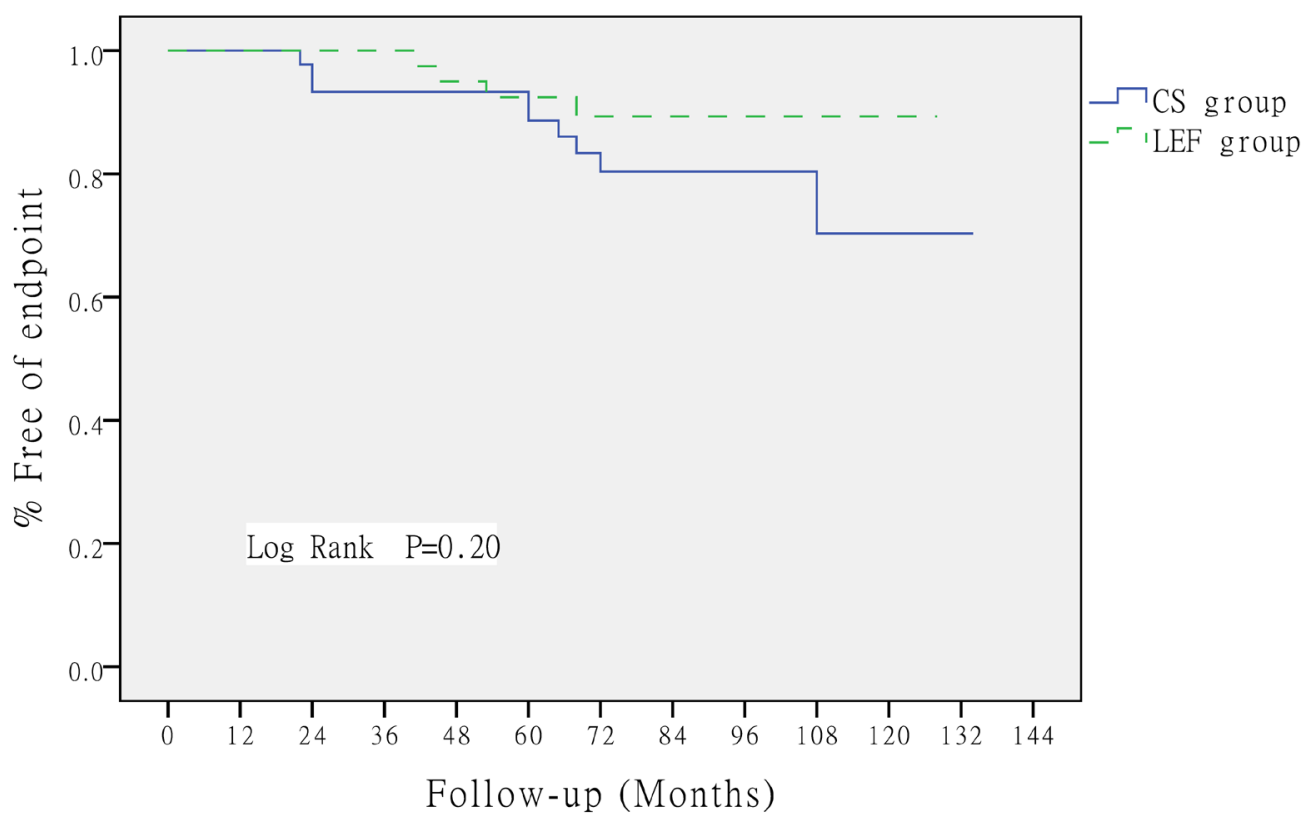

Figure 2: Kaplan-Meier survival for primary outcomes; Log rank significance for ESRD a. and for a $50 \%$ increase in SCr b. 
Table 2: Outcomes of Treatment

\begin{tabular}{|c|c|c|c|c|}
\hline & & CS group $(n=45)$ & LEF group $(n=40)$ & $p$ value \\
\hline \multirow{3}{*}{$\begin{array}{l}\text { UPE } \\
(\mathrm{g} / 24 \mathrm{~h})\end{array}$} & baseline & $2.16(1.36,3.5)$ & $1.94(1.21,2.87)$ & 0.372 \\
\hline & Month 6 & $0.83(0.32,1.94)^{* *}$ & $0.54(0.15,1.15)^{* *}$ & 0.070 \\
\hline & Month 12 & $0.65(0.17,1.46)^{* *}$ & $0.29(0.08,0.69)^{* *}$ & 0.022 \\
\hline \multirow{3}{*}{$\mathrm{SCr}(\mu \mathrm{mol} / \mathrm{L})$} & baseline & $95.40 \pm 32.27$ & $92.59 \pm 35.02$ & 0.712 \\
\hline & Month 6 & $94.46 \pm 35.31$ & $87.75 \pm 32.06$ & 0.379 \\
\hline & Month 12 & $96.24 \pm 38.53$ & $87.66 \pm 32.87$ & 0.292 \\
\hline \multirow{3}{*}{$\operatorname{eGFR}\left(\mathrm{ml} / \mathrm{min} / 1.73 \mathrm{~m}^{2}\right)$} & baseline & $83.64 \pm 29.51$ & $84.17 \pm 26.03$ & 0.933 \\
\hline & Month 6 & $85.32 \pm 30.80$ & $87.79 \pm 26.01$ & 0.702 \\
\hline & Month 12 & $83.74 \pm 31.54$ & $87.51 \pm 27.66$ & 0.575 \\
\hline
\end{tabular}

$* P<0.05$ versus baseline value

$* * P<0.01$ versus baseline value

UPE: urine protein excretion

Scr: serum creatinine

eGFR: estimated glomerular filtration rate

Table 3: Adverse events during the treatment period

\begin{tabular}{|l|c|c|}
\hline & LEF group $(\boldsymbol{n}=\mathbf{4 0})$ & CS group $(\boldsymbol{n}=\mathbf{4 5})$ \\
\hline Hepatotoxicity & 3 & 2 \\
\hline Upper respiratory infection & 4 & 4 \\
\hline Pulmonary infection & 2 & 1 \\
\hline Diarrhea & 1 & 0 \\
\hline Herpes-zoster virus infection & 0 & 2 \\
\hline Pruritus & 1 & 0 \\
\hline Insomnia & 0 & 2 \\
\hline Alopecia & 1 & 0 \\
\hline Abnormal glucose metabolism & 0 & 2 \\
\hline Total $(\mathrm{n})$ & 12 & 13 \\
\hline
\end{tabular}

\section{Blood pressure control and RAS blockade}

During the follow-up period, the patients' blood pressures were stable and similar between the two groups $(\mathrm{SBP}=121.91 \pm 15.03 \mathrm{mmHg}$ for CS $v s .121 .51 \pm 14.58$ $\mathrm{mmHg}$ for LEF, $p=0.78$; DBP $=79.18 \pm 10.69 \mathrm{mmHg}$ for CS vs. $78.34 \pm 9.45 \mathrm{mmHg}$ for LEF, $p=0.43$ ). Thirty-two patients $(71.1 \%)$ in the CS group and 30 patients $(75 \%)$ in the LEF group were treated with ACEI/ARB at the study entry. Three patients in the CS group and 2 in the LEF group received combined antihypertensive regimens at the beginning of the study. During the follow-up period, 6 patients in the CS group and 3 in the LEF group were given other antihypertensive drugs because their blood pressure did not achieve target levels.

\section{Compliance and adverse events}

Table 3 lists the adverse events that occurred during treatments. Five of the 85 patients did not complete the 12-month therapy; 3 of them ( 1 in the CS group and 2 in the LEF group) were lost to follow-up and the other 2 (1 in each group) had serious pulmonary infection.
In the LEF group, 12 of 40 patients (30\%) suffered from adverse events including hepatotoxicity $(n=3)$, upper respiratory infection $(n=4)$, pulmonary infection $(n=2)$, diarrhea $(n=1)$, pruritus $(n=1)$, and alopecia $(n$ $=1$ ).

In the CS group, 13 of 45 patients (28.9\%) suffered from adverse events including hepatotoxicity $(n=2)$, upper respiratory infection $(n=4)$, and pulmonary infection $(n=1)$. Other adverse effects such as herpeszoster virus infection $(n=2)$, insomnia $(n=2)$, and abnormal glucose metabolism $(n=2)$ were only observed in the CS group.

No patients died during the follow-up. In the CS group, two serious adverse events were reported: one patient developed cerebral hemorrhage and diabetes, and another developed aortic dissection. No serious adverse events occurred in the LEF group.

\section{DISCUSSION}

As treatment options for IgAN, a leading cause of primary glomerulonephritis, remain limited, there is a pressing need for improved therapies to alleviate this condition. The present results suggest that LEF plus 
low-dose CS is at least as effective as full-dose steroid monotherapy for the treatment of progressive IgAN, and is associated with both greater reduction of proteinuria during long-term follow up (12-88 months) and the absence of severe adverse events.

Consistent with our findings, previous investigations revealed that LEF could attenuate inflammation and ameliorate kidney injury [25-26]. Although some of the data on the effects of combined immunosuppressive medications plus steroids, as compared to steroids alone, on the remission rate of proteinuria are inconsistent [17] [30], several small clinical trials suggested that LEF can reduce proteinuria and delay IgAN progression [22-24]. A recent meta-analysis [27] of 13 RCTs involving 623 patients compared LEF (alone or plus steroid) with steroid therapy alone. LEF demonstrated a marked advantage on CR/PR of proteinuria, as compared with steroid therapy alone (CR/PR; RR, 2.64; 95\% CI, 1.80-3.86; $P$ $<0.00001)$. Although in our study the remission rate was similar between the LEF and CS groups, proteinuria levels were markedly lower in the LEF group during the followup period. On the other hand, the relapse rates in our trial, i.e. $16.1 \%$ in the CS group and $14.8 \%$ in the LEF group, were also similar between groups and comparable to those reported by Yuan et al. [28].

We observed no statistical differences regarding renal survival between the two groups. Consistent with our study, Pozzi et al. [8] added low-dose azathioprine to CS for IgAN treatment and found that the 5-year renal survival rate was no different than that achieved with steroids alone [29]. However, Tang et al. [11] reported that the 6-year renal survival with mycophenolate mofetil treatment was higher than that with conventional treatment with CS. These studies suggest that the use of immunosuppressive medications alone or in combination with CS for the treatment of IgA nephropathy results in equal or better long-term outcomes than conventional steroid monotherapy.

Most combination therapies with immunosuppressive medications cause considerable side

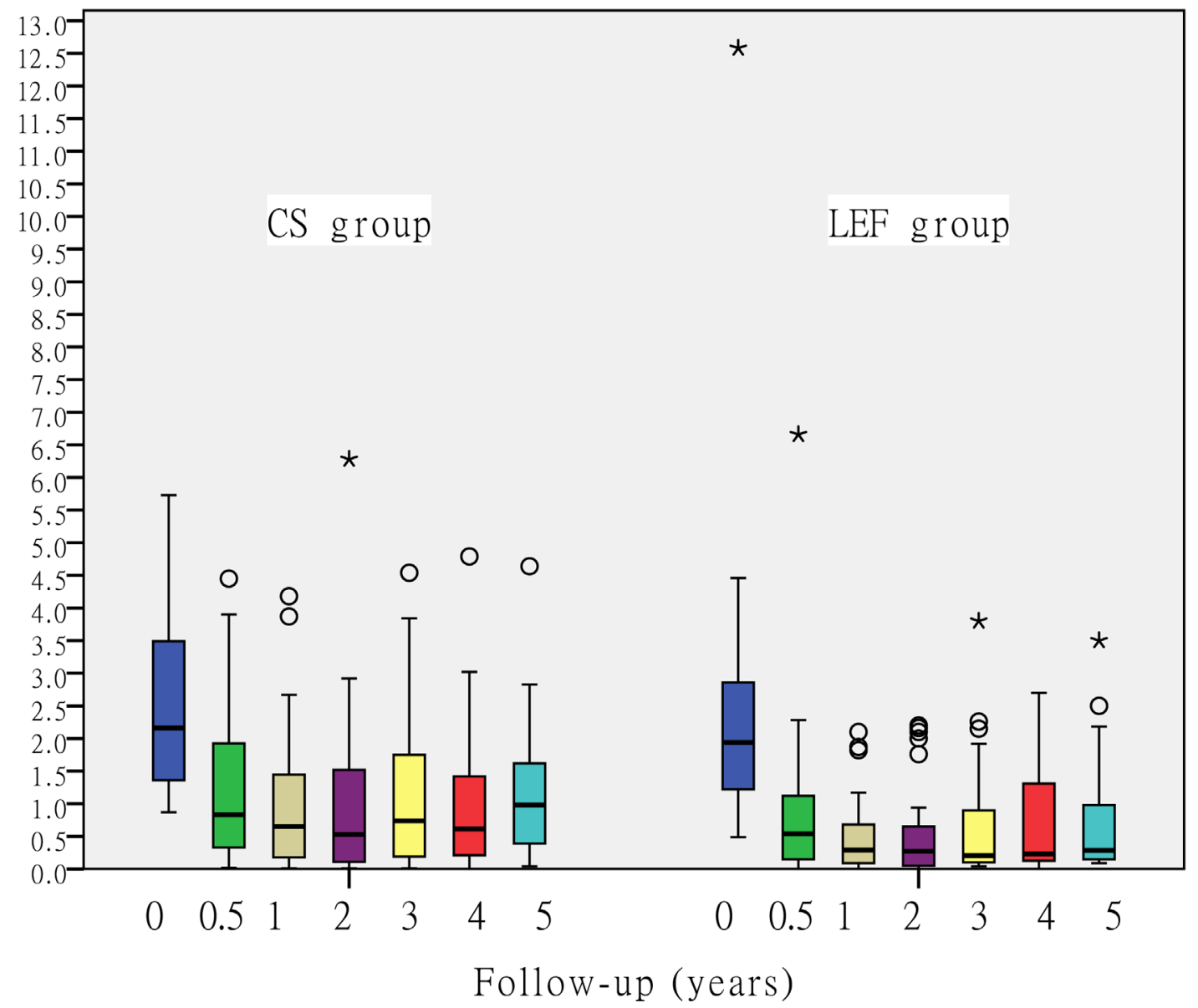

Figure 3: Changes in 24-hour proteinuria over time. In both groups the proteinuria decreased significantly from baseline and no rebound was observed during the follow-up period. (Significantly lower urine protein excretion rates were observed at 24,36 , and 60 months in the LEF group) Significantly lower urine protein excretion rates were observed at 24, 36, and 60 months in the LEF group $(P<$ 0.05; Wilcoxon-Mann-Whitney test). The lines crossing the boxes indicate the median; boxes indicate the IQR, i,e. the spread of the middle $50 \%$ of the values; the whiskers show the largest and smallest observed values that are $<1.5$ box lengths from the 25 th or 75 th percentile. Circles and asterisks indicate more extreme values. 
effects. The adverse events of LEF in IgAN patients were summarized in a meta-analysis [27]. It showed that $4.7 \%$ of patients demonstrated elevated liver enzymes, 3.7\% of patients exhibited digestive symptoms, and $4.4 \%$ patients had alopecia. In our study, the incidence of adverse events was similar between the CS and LEF groups. However, two severe adverse events occurred in the CS group during the follow-up period. Cerebral hemorrhage occurred in a 63-year-old male patient who developed abnormal glucose metabolism in the first round of prednisone and whose blood pressure control was not ideal. Aortic dissection occurred in a 35-year-old male patient with normal blood pressure. These two patients had a history of more than two cycles of CS therapy during the entire treatment and follow-up period, and it is possible that these adverse events were associated with long-term CS use. Our results suggest that LEF combined with low-dose CS is probably safer than full-dose steroid course for the treatment of IgAN, especially in those patients who could not tolerate full therapeutic CS dosages.

There were several limitations in our study. This is a single-center study with a relatively small sample size. Therefore, we could not perform subgroup analysis and true differences in renal survival between the groups might thus be masked. In addition, we could not perform intention-to-treat analysis, which is based on the initial treatment assignment and not on the treatment eventually received, because we could not access the clinical data of 5 patients who were withdrawn from the study. Finally, we did not have a detailed record of the frequency and treatment regimens of the disease relapse cases during the 88-month follow-up period.

In conclusion, a 12-month course of LEF combined with low-dose CS seems to be at least as effective as fulldose CS for the treatment of progressive IgAN, and is associated with both greater reduction of proteinuria and fewer severe adverse events during long-term follow-up. This regimen could be an alternative treatment option for IgAN patients with relative contraindications to full-dose corticosteroid therapy.

\section{MATERIALS AND METHODS}

\section{Patients}

This randomized, controlled, prospective, openlabel, single-center trial enrolled patients from June 1, 2004 to June 30, 2010 at Ren Ji Hospital in Shanghai, China. All patients had biopsy-proven primary IgAN with renal biopsy samples examined independently by two pathologists. Patients aged 18-65 years were included if they had proteinuria $\geq 1.0 \mathrm{~g} / 24 \mathrm{~h}$ and an estimated glomerular filtration rate $($ eGFR) $\geq 30 \mathrm{ml} /$

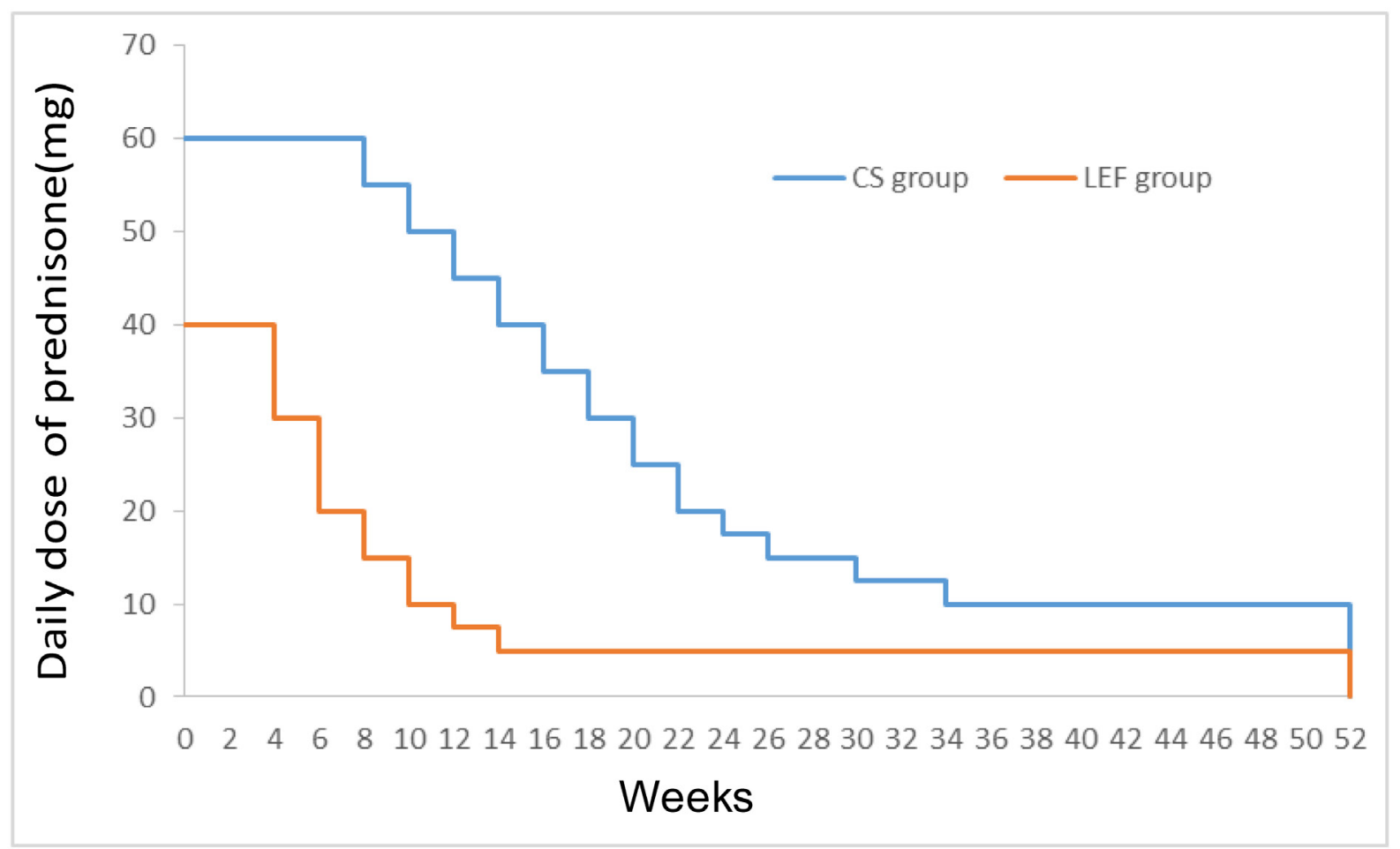

Figure 4: Plot of daily dose of prednisone-time profiles during 52-weeks follow-up for the CS and LEF groups. CS group $=$ full-dose corticosteroids alone; LEF group $=$ leflunomide combined with low-dose of corticosteroids. 
$\min / 1.73 \mathrm{~m}^{2}$ (calculated by CKD-EPI equation). Patients with any of the following conditions were excluded: (i) rapidly progressive IgA nephropathy (IgAN with rapid decline in renal function characterized histologically by necrotizing capillaritis or $>50 \%$ active crescents on biopsy) (ii) secondary IgA nephropathy due to systemic diseases such as Henoch-Schönlein purpura nephritis, hepatitis-associated nephritis, lupus nephritis, etc.; (iii) use of CS or other immunosuppressive agents within 6 months prior to randomization; (iv) serum creatinine (Scr) $>$ 250umol/L; (v) severe infections; (vi) hepatitis B virus carriers and other chronic liver diseases; (vii) presence of malignancy, HIV infection, or acute central nervous system diseases; (viii) abnormal glucose metabolism; (ix) pregnancy or lactation; (x) poor compliance or allergy to study drugs. The study was reviewed and approved by the Ethics Committee at Ren Ji Hospital of Shanghai Jiao tong University Medical School (2002HL0133) and informed consent was obtained from all the participants.

\section{Treatment regimen and evaluations}

Patients were randomized to either leflunomide plus low-dose corticosteroid (LEF group) or to full-dose corticosteroid alone (CS group). Patients in the LEF group were treated with leflunomide $40 \mathrm{mg}$ per day for 3 days, after which the dose was reduced to $20 \mathrm{mg}$ per day and administered for 12 months, in conjunction with oral prednisone, $0.8 \mathrm{mg} / \mathrm{kg} /$ day for $4-6$ weeks. The maximum daily dose of prednisone was $40 \mathrm{mg}$. Then, prednisone was gradually tapered by 10,5 , and $2.5 \mathrm{mg}$ to a maintenance dose of $5 \mathrm{mg}$ per day. Patients in the CS group were treated with oral prednisone $1.0 \mathrm{mg} / \mathrm{kg} /$ day for 8-12 weeks, with a maximum daily dose of $60 \mathrm{mg}$. Then, the daily dose was tapered by 5 and $2.5 \mathrm{mg}$ to a maintenance dose of $10 \mathrm{mg}$ per day. The steroid regimen is illustrated in Figure 4.

No other immunosuppressive or cytotoxic agents were used. All patients were given ACEI/ARB unless blood pressure was $\leq 90 / 60 \mathrm{mmHg}$ or if there was hypotension. Additional anti-hypertensive medications were also prescribed if blood pressure was $>130 / 80$ $\mathrm{mmHg}$ with ACEI/ARB alone. Patients who developed hyperlipidemia, infection, or a hypercoagulable state were treated accordingly.

The duration of the treatment was 12 months. We evaluated clinical efficacy and monitored adverse events at 6- and 12-month time points. After one year of treatment, we continued to follow the patients for a median period of 88 months. We recorded the patients' medications, clinical characteristics, and laboratory results at each follow-up visit. Urinary protein excretion was measured in 24-hour samples obtained at least once a year. During the followup period, the patients were retreated with the original regimen if the disease relapsed.

The criteria for the discontinuation of treatment included severe infection or other serious adverse reactions associated with the tested drugs, pregnancy, and death or lost to follow-up.

The primary outcome was ESRD or a $50 \%$ increase in baseline serum creatinine ( $\mathrm{SCr}$ ). The secondary outcome was changes in proteinuria over time.

Complete remission (CR) was defined as a urine protein excretion (UPE) $<0.3 \mathrm{~g} / \mathrm{d}$ with stable Scr (defined as a change in $\mathrm{SCr}$ of $15 \%$ or less above baseline values). Partial remission (PR) was defined as having at least a $50 \%$ reduction in UPE compared with baseline or 0.3 $\mathrm{g} / \mathrm{d} \leq \mathrm{UPE}<3.5 \mathrm{~g} / \mathrm{d}$ with stable Scr. No response (NR) was defined as a UPE $>3.5 \mathrm{~g} / \mathrm{d}$, or a $<50 \%$ reduction in UPE with or without renal deterioration [31]. Relapse was defined as the reappearance of significant proteinuria, defined as $>1.0 \mathrm{~g} / \mathrm{d}$ and as a UPE increase of $>50 \%$ from the lowest level of proteinuria after remission [31-32].

\section{Renal biopsy}

The following histopathological criteria were recorded: 1) percentage of segmental or global glomerulosclerosis/total glomeruli, 2) percentage of crescentic glomeruli/ total glomeruli, 3) extent (percentage of the biopsied area) of inflammatory cell infiltration $(0=$ absent, $1=1$ to $24 \%, 2=25$ to $50 \%, 3=$ more than $50 \%$ ), 4) extent (percentage of the biopsied area) of interstitial fibrosis and chronic inflammation $(0=$ no fibrosis or inflammation, $1=1$ to $24 \%, 2=25$ to $50 \%, 3=$ more than $50 \%$ ), 5) extent of tubular atrophy (percentage of atrophic tubules; $0=$ no atrophy, $1=1$ to $24 \%, 2=25$ to $50 \%, 3=$ more than 50\%).

\section{Statistical analysis}

Normally distributed variables were expressed as means $\pm \mathrm{SD}$ and compared using a $t$-test or analysis of variance (ANOVA) as required. Non-parametric variables were expressed as median with range and compared using either the Mann-Whitney U test or the Kruskal-Wallis test. The chi-square test was employed for the categorical variables. Kaplan-Meier survival functions with the logrank test were used to calculate the cumulative rates of the primary outcomes in the two groups. All tests were two-tailed, with $p$-values $<0.05$ considered statistically significant. All statistics were done using SPSS 19.0.

\section{Abbreviations}

ACEI, angiotensin-converting enzyme inhibitor; ALB, serum albumin; ALT, alanine transaminase; ARB, angiotensin receptor blocker; $\mathrm{BUN}$, blood urea nitrogen; AST: aspartate aminotransferase; CKD, chronic kidney disease; CR, complete remission; CS, corticosteroids; DBP, diastolic blood pressure; eGFR, estimated 
glomerular filtration rate; ESRD, end-stage renal disease; GLU, glucose; GN, glomerulonephritis; Hb, hemoglobin; IgAN, IgA nephropathy; IQR, interquartile range; LDL, low density lipoprotein; LEF, leflunomide; NR, no response; PR, partial remission; SBP, systolic blood pressure; SD, standard deviation; $\mathrm{SCr}$, serum creatinine; TC, total cholesterol; TG, triglycerides; UA, serum uric acid; UPE, 24-hour urinary protein excretion.

\section{Author contributions}

Zhaohui Ni and Jiaqi Qian conceived and designed the study; Lulin Min analyzed data and wrote the manuscript; Qin Wang, Liou Cao, and Wenyan Zhou performed the study and collected the study data; Jiangzi Yuan, Minfang Zhang, Xiajing Che, Shan Mou, Huili Dai, Wei Fang, Leyi Gu, Mingli Zhu, Ling Wang, and Zanze Yu participated in collection, analysis, and interpretation of the data. Zhaohui Ni revised the manuscript. All authors read and approved the manuscript.

\section{ACKNOWLEDGMENTS}

We are grateful to the National Natural Science Foundation of China and the Shanghai Health Bureau for the financial support. We thank all the doctors at the Nephrology Department of Ren Ji Hospital in Shanghai, China, for their work.

\section{CONFLICTS OF INTEREST}

No potential conflicts of interest were disclosed.

\section{FUNDING}

This study was supported by the National Natural Science Foundation of China (81370794 and 81570604) as well as by a program from the Shanghai Health Bureau (No. ZHYY-ZXYJHZX-1-02).

\section{REFERENCES}

1. Woo KT, Chan CM, Chin YM, Choong HL, Tan HK, Foo M, Anantharaman V, Lee GS, Chiang GS, Tan PH, Lim $\mathrm{CH}$, Tan CC, Lee E, et al. Global evolutionary trend of the prevalence of primary glomerulonephritis over the past three decades. Nephron Clinical practice. 2010; 116:c337346.

2. McGrogan A, Franssen CF, de Vries CS. The incidence of primary glomerulonephritis worldwide: a systematic review of the literature. Nephrology, dialysis, transplantation. 2011; 26:414-430.

3. D'Amico G. Natural history of idiopathic IgA nephropathy and factors predictive of disease outcome. Seminars in nephrology. 2004; 24:179-196.

4. Schrier R. KDIGO Clinical Practice Guideline for Acute Kidney Injury audioicon. 2012.

5 Ballardie FW, Roberts IS. Controlled prospective trial of prednisolone and cytotoxics in progressive IgA nephropathy. Journal of the American Society of Nephrology. 2002; 13:142-148.

6. Jiang XY, Mo Y, Sun LZ, Yue ZH, Chen SM, Wu W. Efficacy of methylprednisolone, cyclophosphamide in pediatric IgA nephropathy assessed by renal biopsy. Clinical nephrology. 2009; 71:625-631.

7. Pozzi C, Andrulli S, Pani A, Scaini P, Roccatello D, Fogazzi G, Pecchini P, Rustichelli R, Finocchiaro P, Del Vecchio L, Locatelli F. IgA nephropathy with severe chronic renal failure: a randomized controlled trial of corticosteroids and azathioprine. Journal of nephrology. 2013; 26:86-93.

8. Pozzi C, Andrulli S, Pani A, Scaini P, Del Vecchio L, Fogazzi G, Vogt B, De Cristofaro V, Allegri L, Cirami L, Procaccini AD, Locatelli F. Addition of azathioprine to corticosteroids does not benefit patients with IgA nephropathy. Journal of the American Society of Nephrology. 2010; 21:1783-1790.

9. Rasche FM, Keller F, Rasche WG, Schiekofer S, Kahn T, Fahnert J. Sequential therapy with cyclophosphamide and mycophenolic acid in patients with progressive immunoglobulin A nephropathy: a long-term follow-up. Clinical and experimental immunology. 2016; 183:307-316.

10. Tang S, Leung JC, Chan LY, Lui YH, Tang CS, Kan CH, Ho YW, Lai KN. Mycophenolate mofetil alleviates persistent proteinuria in $\operatorname{IgA}$ nephropathy. Kidney international. 2005; 68:802-812.

11. Tang SC, Tang AW, Wong SS, Leung JC, Ho YW, Lai KN. Long-term study of mycophenolate mofetil treatment in IgA nephropathy. Kidney international. 2010; 77:543-549.

12. Maes BD, Oyen R, Claes K, Evenepoel P, Kuypers D, Vanwalleghem J, Van Damme B, Vanrenterghem YF. Mycophenolate mofetil in IgA nephropathy: results of a 3 -year prospective placebo-controlled randomized study. Kidney international. 2004; 65:1842-1849.

13. Frisch G, Lin J, Rosenstock J, Markowitz G, D’Agati V, Radhakrishnan J, Preddie D, Crew J, Valeri A, Appel G. Mycophenolate mofetil (MMF) vs placebo in patients with moderately advanced IgA nephropathy: a doubleblind randomized controlled trial. Nephrology, dialysis, transplantation. 2005; 20:2139-2145.

14. Chen Y, Li Y, Yang S, Li Y, Liang M. Efficacy and safety of mycophenolate mofetil treatment in IgA nephropathy: a systematic review. BMC nephrology. 2014; 15:193.

15. Kim YC, Chin HJ, Koo HS, Kim S. Tacrolimus decreases albuminuria in patients with IgA nephropathy and normal blood pressure: a double-blind randomized controlled trial of efficacy of tacrolimus on IgA nephropathy. PloS one. 2013; 8:e71545.

16. Masutani K, Tsuchimoto A, Yamada T, Hirakawa M, 
Mitsuiki K, Katafuchi R, Hirakata H, Kitazono T, Tsuruya $\mathrm{K}$. Comparison of steroid-pulse therapy and combined with mizoribine in IgA nephropathy: a randomized controlled trial. Clinical and experimental nephrology. 2016; 20:896903.

17. Vecchio M, Bonerba B, Palmer SC, Craig JC, Ruospo M, Samuels JA, Molony DA, Schena FP, Strippoli GF. Immunosuppressive agents for treating IgA nephropathy. The Cochrane database of systematic reviews. 2015; 8: Cd003965.

18. Smolen JS, Landewe R, Breedveld FC, Buch M, Burmester G, Dougados M, Emery P, Gaujoux-Viala C, Gossec L, Nam J, Ramiro S, Winthrop K, de Wit M, et al. EULAR recommendations for the management of rheumatoid arthritis with synthetic and biological disease-modifying antirheumatic drugs: 2013 update. Annals of the rheumatic diseases. 2014; 73:492-509.

19. Cao H, Rao Y, Liu L, Lin J, Yang H, Zhang X, Chen Z. The Efficacy and Safety of Leflunomide for the Treatment of Lupus Nephritis in Chinese Patients: Systematic Review and Meta-Analysis. PloS one. 2015; 10:e0144548.

20. Zhang Y, Gao Y, Zhang Z, Liu G, He H, Liu L. Leflunomide in addition to steroids improves proteinuria and renal function in adult Henoch-Schoenlein nephritis with nephrotic proteinuria. Nephrology (Carlton, Vic). 2014; 19:94-100.

21. Yang S, Xie L, Xue W, Yin A, Lu W. Leflunomide plus oral prednisone in treatment of idiopathic membranous nephropathy: a retrospective clinical study of efficacy and safety. Nephrology (Carlton, Vic). 2013; 18:615-622.

22. Lou T, Wang C, Chen Z, Shi C, Tang H, Liu X, Yin P, $\mathrm{Yu} X$. Randomised controlled trial of leflunomide in the treatment of immunoglobulin A nephropathy. Nephrology (Carlton, Vic). 2006; 11:113-116.

23. Cheng G, Liu D, Margetts P, Liu L, Zhao Z, Liu Z, Tang L, Fang Y, Li H, Guo Y, Chen F, Liu F. Valsartan combined with clopidogrel and/or leflunomide for the treatment of progressive immunoglobulin A nephropathy. Nephrology (Carlton, Vic). 2015; 20:77-84.

24. Wu J, Duan SW, Sun XF, Li WG, Wang YP, Liu WH, Zhang JR, Lun LD, Li XM, Zhou CH, Li JJ, Liu SW, Xie YS, et al. Efficacy of Leflunomide, Telmisartan, and Clopidogrel for Immunoglobulin A Nephropathy: A Randomized Controlled Trial. Chinese medical journal. 2016; 129:1894-1903.
25. Yu WM, Wang H, Ren XJ, Liu JP, Wang JY. Experimental study of leflunomide on renal protective effect and on inflammatory response of streptozotocin induced diabetic rats. Nephrology (Carlton, Vic). 2012; 17:380-389.

26. Bloudickova S, Rajnoch J, Lodererova A, Honsova E, Viklicky O. Leflunomide derivate FK 778 in accelerated renal injury in transgenic rat. Folia biologica. 2010; 56:7277.

27. Liu Y, Xiao J, Shi X, Hao G, Chen Q, Zhou J, Wei X. Immunosuppressive agents versus steroids in the treatment of IgA nephropathy-induced proteinuria: A meta-analysis. Experimental and therapeutic medicine. 2016; 11:49-56.

28. Yuan Y, Che X, Ni Z, Zhong Y, Qi Y, Shao X, Wang Q, Cao L, Zhang M, Xie Y, Qi C, Tian L, Mou S. Association of Relapse with Renal Outcomes under the Current Therapy Regimen for IgA Nephropathy: A Multi-Center Study. PloS one. 2015; 10:e0137870.

29. Pozzi C, Bolasco PG, Fogazzi GB, Andrulli S, Altieri P, Ponticelli C, Locatelli F. Corticosteroids in IgA nephropathy: a randomised controlled trial. Lancet (London, England). 1999; 353:883-887.

30. Yoshikawa N, Honda M, Iijima K, Awazu M, Hattori S, Nakanishi K, Ito H. Steroid treatment for severe childhood IgA nephropathy: a randomized, controlled trial. Clinical journal of the American Society of Nephrology. 2006; 1:511-517.

31. Kim JK, Kim JH, Lee SC, Kang EW, Chang TI, Moon SJ, Yoon SY, Yoo TH, Kang SW, Choi KH, Han DS, Kie JH, Lim BJ, et al. Clinical features and outcomes of IgA nephropathy with nephrotic syndrome. Clinical journal of the American Society of Nephrology. 2012; 7:427-436.

32. Moon SJ, Park HS, Kwok SK, Ju J, Choi BS, Park KS, Min JK, Kim HY, Park SH. Predictors of renal relapse in Korean patients with lupus nephritis who achieved remission six months following induction therapy. Lupus. 2013; 22:527537. 